\title{
REVIEW ARTICLE The development of neonatal neurointensive care
}

\author{
Topun Austin (1D)
}

Brain injury remains one of the major unsolved problems in neonatal care, with survivors at high risk of lifelong neurodisability. It is unlikely that a single intervention can ameliorate neonatal brain injury, given the complex interaction between pathological processes, developmental trajectory, genetic susceptibility, and environmental influences. However, a coordinated, interdisciplinary approach to understand the root cause enables early detection, and diagnosis with enhanced clinical care offering the best chance of improving outcomes and facilitate new lines of neuroprotective treatments. Adult neurointensive care has existed as a speciality in its own right for over 20 years; however, it is only recently that large prospective studies have demonstrated the benefit of this model of care. The 'Neuro-intensive Care Nursery' model originated at the University of California San Francisco in 2008, and since then a growing number of units worldwide have adopted this approach. As well as providing consistent coordinated care for infants from a multidisciplinary team, it provides opportunities for specialist education and training in neonatal neurology, neuromonitoring, neuroimaging and nursing. This review outlines the origins of brain-oriented care of the neonate and the development of the NeuroNICU (neonatal intensive care unit) and discusses some of the challenges and opportunities in expanding this model of care.

Pediatric Research _\#\#\#\#\#\#\#\#\#\#\#\#\#\#\#\#\#\#_; https://doi.org/10.1038/s41390-019-0729-5

\section{INTRODUCTION}

Brain injury can affect both preterm and term infants; the most common disorders are neonatal encephalopathy (NE) and perinatal arterial ischaemic stroke (PAIS) in term infants and the consequences of germinal matrix-intraventricular haemorrhage (GMH-IVH) and white matter injury (WMI) in extremely low birthweight preterm infants. Metabolic and rare genetic disorders can also present with seizures and encephalopathy in the neonatal period. All these vulnerable infants are at significant risk to develop cerebral palsy, epilepsy and other forms of neurological disability. 'These outcomes place an enormous physical, psychological and financial burden on individuals, families and society; for example, costs of preterm birth to the public sector in the United Kingdom is estimated at over $£ 2.9$ billion/year; ${ }^{2}$ the additional lifetime cost of looking after a child with cerebral palsy in the United States can be over one million dollars. ${ }^{3}$

The past 40 years have seen a dramatic improvement in the survival of extremely preterm and critically ill neonates; however, the incidence of cerebral palsy and other neurodevelopmental problems remains high. ${ }^{4,5}$ The global scale of perinatal brain injury cannot be underestimated: in 2015 an estimated 690,000 infants died from intrapartum-related events, and just over 1 million infants died from complications of prematurity. ${ }^{6}$ Therapeutic hypothermia represents the first neuroprotective therapy for infants with $\mathrm{NE}$, yet even with hypothermia, over half treated infants are at risk for moderate-severe cerebral palsy or death. ${ }^{7}$ No single intervention can ameliorate neonatal brain injury, given the multiple pathologies, complex interaction between pathological processes, developmental trajectory, genetic susceptibility and environmental influences. But a coordinated, high-expertise approach to enable early detection and diagnosis and with enhanced clinical care could offer the best chance of improving outcomes as well as facilitate new lines of neuroprotective research. It is this philosophy that has motivated clinicians in developing the neonatal neurocritical care (NNCC) model of service delivery, which over the past 10 years has gained considerable traction in North America and is being increasingly adopted across the world.

This article examines the evidence from adult medicine that a dedicated neurointensive care approach can improve outcomes, reviews the development of neonatal neurointensive care and addresses what some of the challenges and benefits of this model of care might be.

\section{ADULT NEUROINTENSIVE CARE: WHAT CAN WE LEARN?}

'Neurocritical care is devoted to the comprehensive care of critically ill patients with neurological or neurosurgical disease. Care of such patients requires an understanding of the physiology and pathophysiology common to brain diseases in general, as well as the skills and knowledge to treat a range of specific conditions. Given the exquisite vulnerability of the injured brain to physiological insults, optimal care of such patients demands meticulous attention to maintenance of systemic and cerebral physiological targets while ensuring appropriate protection of extra-cranial organs. ${ }^{8}$

This is the definition of adult neurocritical care given by the UK Intensive Care Society, but could equally be applied to a definition of neonatal neurocritical care. Adult neurocritical care has been recognised as a subspecialty in its own right for almost 20 years. Its origins can be traced back to specialist critical care units developing within neuroscience centres focusing on patients with traumatic brain injury (TBI) and subarachnoid haemorrhage (SAH); today large dedicated neurocritical care units care for patients with a wide range of neurological and neurosurgical conditions. A key question with the emergence of a new subspecialty is whether

\footnotetext{
${ }^{1}$ Neonatal Intensive Care Unit, Cambridge University Hospitals NHS Foundation Trust, Cambridge, UK

Correspondence: Topun Austin (ta338@cam.ac.uk)
}

Received: 2 September 2019 Revised: 6 December 2019 Accepted: 9 December 2019

Published online: 18 December 2019 
it improves outcomes? In this respect, improvement science has lagged behind other areas of clinical research with most of the evidence coming from single centre studies using retrospective data and historical controls; only a few multicentre prospectively controlled studies have been carried out. Most of the evidence relates to TBI. Data from the Trauma Audit and Research Network showed that outcome for patients managed in a general hospital were worse than those cared in a neuroscience centre $(2.15$-fold increase (95\% confidence interval $(\mathrm{Cl}): 1.77-2.60)$ in the odds of death adjusted for case mix). ${ }^{9}$ A large multicentre observational study, Risk Adjustment In Neurocritical care (RAIN) confirmed better outcomes in neuroscience centres. ${ }^{10}$ Similar improved outcomes have been reported in patients with intra-cerebral haemorrhage and SAH. ${ }^{11,12}$

As well as the methodological limitations outline above, another issue is that the outcome data is primarily mortality and short-term outcome, with little in the way of long-term neurological outcome studies. If we accept that specialist neurocritical care units improve outcomes, a perhaps more important question is why? Given the complexity of the specialty and heterogeneity of the patients, there is no simple answer. However, a number of factors, highlighted by Tweedie, include specific experience and increased caseload of multidisciplinary teams, brain centred care and specialist neuromonitoring, rapid neurosurgical access and early input of physiotherapy and neurorehabilitation, and a less nihilistic attitude and more conservative approach to the end of life. ${ }^{13}$

Outside the neurocritical care unit, the development of specialised stroke units has revolutionised the management of adult stroke. ${ }^{14}$ A Cochrane review in 2013, analysing 28 trials enroling 5855 patients concluded that stroke patients who received organised inpatient care in a stroke unit were more likely to be alive, independent, and living at home 1 year after the stroke. As with neurocritical care, the focus of the stroke unit is to provide consistent specialised care by a multidisciplinary team. While a major advance was the alteplase trial in 1995, which demonstrated improved outcome with hyperacute thrombolysis (within $3 \mathrm{~h}$ of treatment), it is the provision of this therapy within the context of a dedicated stroke unit which appears to maximise benefit. ${ }^{15}$ The authors of the Cochrane review acknowledge that their analysis does not explain how these units may improve outcome, but speculate that this could be due to, among other things, greater staff expertise, better diagnostic procedures, better nursing care and more effective rehabilitation procedures. As with many causes of brain injury, rapid and timely intervention is crucial as highlighted by the development of mobile stroke units for prehospital thrombolysis. ${ }^{16}$ In adults, children and neonates timely intervention prior to admission to specialised units is important to prevent lifelong brain injury.

\section{BRAIN INJURY IN THE NEWBORN}

Gestational age remains the most important factor determining the aetiology and consequences of brain injury. In the term infant, NE resulting from a perinatal hypoxic-ischaemia insult is the leading cause of acquired brain injury and neurodisability, with an incidence ranging from 1 to 12 per 1000 live births depending on the precise definition and resource setting. ${ }^{17}$ In many respects this condition most closely resembles adult TBI in that it follows an acute insult, early management is time critical and the goal is to prevent secondary injury by meticulous attention to cerebral and systemic physiology. PAIS is perhaps an under-recognised cause of acquired brain injury: with an incidence of around 2.5 per 1000 live births, it is the second most common cause of neonatal seizures and the most common cause of childhood hemiplegia. ${ }^{18}$ Preterm brain injury is complex and multifactorial. With improvements in intensive care, the incidence of GMH-IVH has fallen in infants > 26 weeks; however, with increased survival, the prevalence remains high. ${ }^{19}$ The spectrum of injury to the periventricular white matter has shifted from the destructive lesions of periventricular leukomalacia to a more subtle reactive WMI. ${ }^{20}$ In the preterm infant, brain injury is not always the primary event, but the consequence of preterm birth and associated comorbidities has a profound impact on brain development. In a large metab-analysis looking at the cognitive outcomes of preterm infants born since 1990, Twilhaar et al. ${ }^{21}$ reported that bronchopulmonary dyplasia was the most significant factor for long-term cognitive outcome. This is why no single intervention is likely to ameliorate preterm brain injury, yet approaches to minimise the impact of prematurity on the infant may be beneficial. A number of childhood neurological conditions also present in the neonatal period, where early recognition and diagnosis could have a significant impact on the disease progression. The most famous condition being phenylketonuria, where universal screening has dramatically altered the prognosis of this condition; ${ }^{22}$ there is emerging evidence that some epileptic encephalopathies, if diagnosed early, may benefit from specific anti-epileptic medication with altered outcome. ${ }^{23}$

\section{STRATEGIES TO PROTECT THE NEWBORN BRAIN}

With improvements in overall survival of newborn infants on intensive care, it was natural that attention would shift to intact survival with good neurodevelopmental outcome. Many groups around the world recognised this, but it is certainly worth mentioning that the NICU (neonatal intensive care unit) at the University College London under the leadership of the late Professor Osmund Reynolds. Not only did he recognise the importance of long-term neurodevelopmental follow-up (the 1979 cohort of infants from UCL are still being studied) ${ }^{24}$ but also the value of the then emerging technologies of cranial ultrasound (CUS) and later magnetic resonance imaging and spectroscopy to understand the pathophysiology of cerebral injury. ${ }^{25,26}$ Work by the Reynolds group and others in the 1990s led to the concept of a delayed, or secondary energy failure following hypoxia-ischaemia. ${ }^{27}$ This directly led to the experimental studies and subsequent clinical trials of therapeutic hypothermia. ${ }^{28}$ Despite equivocal data on the benefit of therapeutic hypothermia following TBI and cardiac arrest in adults and children, ${ }^{29}$ cooling neonates following perinatal asphyxia has shown to be particularly efficacious: the meta-analysis of over 11 clinical trials involving 1505 infants showed therapeutic hypothermia significantly reduced death or disability at 18 months (relative risk $0.75,95 \%$ $\mathrm{Cl} 0.68-0.83$ vs. standard care); the number needed to treat for one newborn baby to be alive and free of disability is 7.7 In 2010 the International Liaison Committee on Resuscitation published guidelines stating:

Newly born infants born at or near-term with evolving moderate to severe hypoxic-ischemic encephalopathy should be offered therapeutic hypothermia...Cooling should be initiated and conducted under clearly defined protocols with treatment in neonatal intensive care facilities and with the capability for multidisciplinary care. ${ }^{30}$

There is often a delay in translating clinical research into the standard of care; this did not seem to be the case though in the UK where therapeutic hypothermia was widely adopted, mainly a result of many NICU's participating in the largest randomised controlled trial for therapeutic hypothermia, the TOtal BodY hypothermia trial (TOBY) study (ISRCTN8954757). ${ }^{31}$ Following completion of that trial, the UK TOBY Cooling Register was set up to provide guidance to clinicians considering the introduction of this therapy, audit the uptake and conduct of therapeutic hypothermia in the United Kingdom and to undertake surveillance 
for adverse effects related to cooling. Reporting the results from 4.5 years of data from the register was published in 2012, providing a unique insight into the uptake of a new therapy in the United Kingdom. ${ }^{32}$ During this period, a total of 2069 infants were registered, which equates to 1-2/1000 live births/year. However, without contemporaneous epidemiological data on the incidence of $\mathrm{NE}$, it remains unclear whether all infants who may benefit from this treatment actually receive it.

The UK experience also benefited from an established regional neonatal network provision of care and neonatal transport infrastructure. For example, in the East of England, the Acute Neonatal Transfer Team began transferring infants for therapeutic hypothermia in October 2009; in the first 18 months, the time taken to achieve target temperature had reduced significantly (regression coefficient $-12.8 ; 95 \% \mathrm{Cl} 19.2$ to $-6.5, p=0.0002$ ). To address the issue of infants arriving at the cooling centre outside the target temperature range, a servo-controlled cooling mattress was installed on the transport incubator, which resulted both in a significant improvement in thermal control and also a reduction in stabilization time. ${ }^{33}$ The practice of active cooling on transport has subsequently been adopted by most transport teams in the United Kingdom.

\section{DEVELOPMENT OF THE NEURO-NICU}

The multidisciplinary approach to the care of the newborn infant with brain injury is not new, with many units working closely with paediatric neurologists, neurosurgeons and other professionals. However, the advent of therapeutic hypothermia represented a step change requiring a coordinated approach to the identification and management of patients with NE. The University of California San Francisco (UCSF) were one of the first units in the United States to set up what they called a neonatal neurointensive care nursery (NICN). ${ }^{34}$ Over the past decade, this approach has gained considerable traction, particularly in North America with a number of units reporting their experience of setting up similar models of care. ${ }^{35,36}$ The goal of the NICN is to utilize the advances in neuromonitoring, imaging with new treatments, which would ultimately improve neurodevelopmental outcomes in newborns at risk of neurological disability. Three key patient groups emerged as potentially benefitting from this management approach: (i) term infants with NE and seizures, (ii) extremely preterm infants and (iii) infants with congenital or rare neurological conditions presenting in the neonatal period. Four key themes have also emerged in the development of this and other NNCC services and are discussed below: (i) co-management of infants, (ii) standardized protocols and care bundles, (iii) increased utilization of neuromonitoring and (iv) development of training programmes.

\section{Co-management of infants}

Central to the Neuro-NICU is the co-management of infants at risk of neurological injury with neonatologists, paediatric neurologists, neurophysiology/epileptologists, neuroradiologists, neurosurgeons and specialist nurses. Historically, neonatal neurology was always seen as the purview of the neonatologist, with paediatric neurologists being called upon later on in the management of complex neurological infants. The major step change was the involvement of the paediatric neurologist from the point of admission or initial diagnosis. This gave a broader perspective to both neonatologist and neurologist in the investigation and management of these infants as well as providing continuity of care for families while in the NICU and through to follow-up following discharge. ${ }^{37}$

\section{Standardized protocols}

The process of standardization is a common practice in many industries and has repeatedly been shown to improve outcomes within healthcare settings. ${ }^{38,39}$ As part of an integrated care pathway or care bundle, treatment protocols enable consistency of care, reduces variability and mitigates against error. The NNCC special interest group (NNCC-SIG), set up in 2016, has a number of protocols from different US centres available to download (www. sites.google.com/view/nnccsig/home). It should be noted though that apart from protocols for therapeutic hypothermia, none has been rigorously validated in multicenter studies with meaningful outcomes, which is important to demonstrate improvement. Nevertheless, increased open access and sharing of protocols is an important first step in this process.

\section{Neuromonitoring and neuroimaging}

The use of CUS and amplitude-integrated electroencephalography (aEEG) has been universal among large NICU's for many years. Interestingly, the use of aEEG was first described in the late 1960s for long-term monitoring of adults on intensive care during status epilepticus or following cardiac arrest. ${ }^{40}$ The first use of aEEG from neonates was published in the early 1980s and early studies revealed the presence of subclinical seizures in infants with NE. ${ }^{41,42}$ It was not until the CoolCap and TOBY trials for therapeutic hypothermia, which coincided with the digitization of instrumentation, that its use to monitor infants with NE and seizures became more commonplace. ${ }^{31,43}$ aEEG has also shown to be of value in infants with congenital heart disease and inborn errors of metabolism. ${ }^{44,45}$ The use of aEEG in preterm infants is increasing both to assess brain maturation and also identify seizures, which are increasingly recognized in this group. ${ }^{46,47}$

Despite its ease of use, aEEG remains a long-term trend monitor and lacks the temporal or spatial resolution to a full montage video EEG (vEEG). However, despite the concept of the NeuroNICU being around for over 10 years now, the use of prolonged continuous vEEG is not universal, with only between 35 and $67 \%$ of NICU's reporting their usage. ${ }^{37}$ This is despite the recommendations from the American Clinical Neurophysiological Society, and may represent the challenges around prolonged monitoring and reporting of full montage EEGs. ${ }^{48}$

Continuous monitoring of cerebral oxygenation using nearinfrared spectroscopy (NIRS) is appealing as it is the nearest clinicians have to a measurement of cerebral perfusion at the cotside. While NIRS has been used extensively as a research tool for many years, it has been slow to translate into clinical practice. ${ }^{49}$ There are a number of patient groups at risk of brain injury who may benefit from NIRS monitoring, including preterm infants (especially at risk of IVH or with a large patent ductus arteriosus), encephalopathic infants and infants with congenital heart disease. Potential reasons for the lack of universal uptake include concerns around 'normal' values, variation between instruments, need for treatment guidelines for when thresholds are reached and whether monitoring improves outcomes. The SafeBoosC consortium made up of NICUs across Europe undertook a phase II randomized controlled trial comparing standard treatment against continuous NIRS monitoring with an evidence-based guideline and demonstrated in the treatment group a significant reduction in the burden of hypoxia. ${ }^{50} \mathrm{~A}$ larger phase III trial is currently underway to see if NIRS monitoring reduces mortality and brain injury as assessed on CUS (NCT03770741).

Following the introduction of CUS on the NICU in the 1980s, early studies showed the prognostic value of both imaging and assessment of post-asphyxial hyperaemia in term infants with NE and seizures. ${ }^{51,52}$ Later studies, however, have shown a more limited role of CUS in predicting outcome. ${ }^{53}$ Today, the use of MRI both from a diagnostic and prognostic perspective is almost universal in these infants. ${ }^{54}$ The value of MR spectroscopy in predicting outcome was highlighted in the study by Lally et al., ${ }^{55}$ which reported that thalamic proton spectroscopy 4-14 days after birth was more predictive of 2-year outcome than both conventional MRI, aEEG and neurological examination. While CUS scanning in preterm infants is almost universal in the early 
neonatal period and is particularly valuable for diagnosing GMHIVH and following up infants with post haemorrhagic ventricular dilatation, CUS is less sensitive at diagnosing non-cystic white matter lesions. The prognostic value of CUS has been extensively investigated, while there are some studies which have shown that CUS at TEA improves prognostication; ${ }^{56}$ there are also studies which demonstrate abnormal developmental outcomes despite normal CUS at TEA and others which demonstrate normal outcomes with abnormal CUS. ${ }^{57,58}$ The practice of MRI scanning preterm infants at term equivalent age (TEA) is not universal and data on its value is conflicting. Woodward et al. ${ }^{59}$ reported that MRI at TEA predicts cognitive delay, motor delay, cerebral palsy and neurosensory impairment at 2 years. More recently the ePRIME study in the United Kingdom reported that although MRI predicted adverse motor outcomes slightly better than CUS, both were insensitive at predicting cognitive outcome at 20 months. ${ }^{60}$ However, WMI is often associated with behavioural abnormalities, which may not become apparent at a later age; in this respect, Iwata et al. ${ }^{61}$ found that, in a cohort of very preterm infants, abnormal white matter appearance on term MRI was associated with cognitive impairment at 9 years of age. Finally, it should be noted that the predictive value of MRI scans is highly dependent on the quality of the images, neuroradiology expertise and also associated clinical co-morbidities.

\section{Development of training programmes}

Recognized early by the UCSF team was the requirement to engage the nursing staff. ${ }^{61}$ The aim was to train a cohort of nurses in what were seen as the 'core competencies' of neonatal neurocritical care. This included an understanding of the common neurological conditions encountered in the NICU with an emphasis on clinical presentation, investigations, treatment protocols and use of $a E E G / E E G$ and NIRS monitoring. Once a critical number of Neuro-NICU nurses had been trained, there would be at least two available on each shift to take a lead for these patients. A growing number of online and in-person training programmes, including certified courses, are now available.

More recently, it has been proposed to develop formal training for medical staff in neonatal neurocritical care. While certification in Critical Care Neurology has been available since the early 2000s, Smyser et al. ${ }^{62}$ argue that there is a strong case for a similar programme in fetal and neonatal neurology, pointing out that many trainees currently undergo personal 'bespoke' training mainly in centres with strong neonatal neurology research credentials.

\section{DOES THE NEURO-NICU IMPROVE OUTCOMES?}

The development of the Neuro-NICU has many similarities with the origin of adult neurocritical care and other evolving specialties. Various models of care have been put forward with similar aims and objectives. Like adult neurocritical care, the challenge is to demonstrate that this model of care improves outcomes. To date, data are limited to single centre studies with only short-term outcomes reported. Perhaps, not surprisingly, the presence of a neonatal neurologist led to an increase in the number of consultations, visits per patients and types of patient seen. ${ }^{63}$ The use of vEEG has been shown to significantly increase the seizure detection rate: an important finding considering the limitations on making a diagnosis on clinical grounds alone. ${ }^{64}$ The use of vEEG has also been shown to result in a modest decrease in the use of anticonvulsants and reduced use of anticonvulsant medication at discharge. ${ }^{65}$ It is unclear why this may be the case, but the authors speculate that it may be due to increased confidence in decision making (including stopping anticonvulsants) with vEEG.

The use of care bundles has been most widely used in the context of sepsis. ${ }^{66}$ Within the Neuro-NICU preventing hospital-acquired infection is highly relevant given the relationship between sepsis, necrotizing entercolitis and neurodevelopmental outcome in the preterm infant. ${ }^{67}$ More recently, the use of care bundles to prevent or minimize the complications of IVH have been reported with promising results. Chiriboga et al. ${ }^{68}$ reported an impressive and sustained reduction of IVH from 24 to $9.7 \%$ through the implementation of a multidisciplinary quality improvement initiative, which included focusing on delivery unit management (delayed cord clamping, optimizing cardiopulmonary resuscitation and improved thermoregulation), as well as a bundle of care on the NICU focusing primarily on minimal handling over the first $72 \mathrm{~h}$ of life.

In 2006, following the completion of the TOBY trial, the largest $\mathrm{RCT}$ for therapeutic hypothermias, a national register was set up to collect data from all participating units in the United Kingdom as therapeutic hypothermia became the standard of care for infants with HIE. ${ }^{32}$ This register proved to be a valuable resource to monitor the uptake of this therapy, report rare adverse events and document 'drift' in inclusion criteria. Although a number of national and international registers exist, the most well known being the Vermont-Oxford Network, none are specifically focused on the Neuro-NICU. The creation of the NNCC-SIG is a welcome development in bringing the community together, but it is important that lessons from adult neurocritical care are learnt and national and international data repositories are created to formally evaluate the benefits from the Neuro-NICU model.

As an example that neonatal neurocritical care extends beyond dedicated 'NeuroNICU's, the PRevention of Cerebral palsy in PreTerm labour (PReCePT) project in the United Kingdom is an initiative to ensure all mothers in threatened preterm labour are given antenatal magnesium sulfate to prevent cerebral palsy in preterm infants, regardless of where they are born. ${ }^{69}$ The initial package of training and support materials was developed in the South West of England and has now been rolled out across all maternity units in England. The UK benefits from an integrated clinical research network, which enables both rapid roll out and nationwide evaluation.

\section{DEVELOPMENTS IN NEONATAL NEUROPROTECTION}

New therapies for HIE

Therapeutic hypothermia for HIE was one of the main drivers in the development of the first NICN at UCSF; while cooling represents one of the most significant developments in neonatal care in recent years, nearly half of infants who receive it will still have an abnormal outcome. ${ }^{6}$ The search for adjunct therapies has moved from preclinical to clinical studies. Although the early promise of Xenon as a neuroprotectant did not appear to translate into clinical benefit, other drugs including erythropoietin and allopurinol are subject to ongoing clinical trials and the results are awaited with interest. ${ }^{70-72}$ The debate as to whether infants with mild HIE may benefit from therapeutic hypothermia is ongoing. ${ }^{73}$ The COoling in Mild EncephalopaThy (COMET) trial is a phase II trial investigating the feasibility and optimal duration of cooling infants with mild encephalopathy (NCT03409770). The challenge in this patient cohort remains the ability to identify early infants who may benefit from therapeutic hypothermia and assess meaningful outcomes in a timely manner.

\section{Precision medicine in the NICU}

A number of rare congenital neurological disorders present in the neonatal period, sometimes with subtle manifestations which can be easily missed. With the advent of rapid whole-exome and -genome sequencing (WGS), it is possible that many conditions can be diagnosed early on enabling more timely management, including in some instance's early treatment, for example, in the case of neonatal seizures caused by KCNQ2 mutation, which can be responsive to carbamazepine therapy. ${ }^{23}$ 
Several groups have reported preliminary findings using rapid WGS in targeted cohorts on the $\mathrm{NICU} ;{ }^{74,75}$ the largest of these studies from Cambridge, UK, enroled a total of 195 families undertaking predominantly trio analysis from both the NICU and Paediatric Intensive Care Unit. ${ }^{76}$ In the neonatal cohort, 113 families were recruited (representing $47 \%$ of those approached and $\sim 10 \%$ total admissions to the NICU) and the resulting diagnostic rate was $13 \%$. Of interest, the phenotypic description of the infant was a poor predictor of the gene identified. The diagnosis affected clinical management in $83 \%$ of the neonatal cases, including modification of treatment and care pathways and/or informing palliative care decisions. Of note, the study was integrated into the clinical NHS pipeline, with meaningful results being turned around within 2-3 weeks.

As the Neuro-NICU develops, it is likely rapid WGS will become a requisite investigation tool for a large number of patients, but in turn will also raise important ethical questions. ${ }^{77}$

\section{Multiparameter 'intelligent' monitoring}

Critical care medicine has always been a data-rich environment, with real-time monitoring of many physiological parameters and frequent investigations. However, it is only recently with the advent of computer algorithms driving the 'big data' revolution that the power of these data can potentially be harnessed in the NICU. The purpose of this approach is multifactorial, including deriving new physiological parameters and indices in real time (e.g. cerebral autoregulation), ${ }^{78}$ aiding clinical diagnosis (e.g. seizures), ${ }^{79}$ reducing false-positive alarms ${ }^{80}$ and predicting early patient deterioration (e.g. sepsis). ${ }^{81}$

Biological systems are inherently complex and interrelated at different levels. However, there is a tendency in the management of the critically ill patient to treat data as discrete, linear and stationary and the inherent complexity within the whole biological system is often overlooked. For example, using a non-linear approach, Sortica da Costa et al. ${ }^{82}$ investigated the complexity of biological signals in preterm infants in the first $24 \mathrm{~h}$ of age using multi-scale entropy and found that reduced complexity of brain oxygenation signals was predicative of mortality and brain injury.

Bioinformatics and artificial intelligence are rapidly developing fields, but their translation into clinical practice within the NeuroNICU will require close collaboration between clinicians, engineers, computer scientists, and bioinformaticians and large-scale multicenter studies to validate the different methodologies.

Global challenges in developing neonatal neurocritical care The global burden of perinatal brain injury cannot be underestimated: approximately a quarter of all newborn deaths result from NE, with $96 \%$ occurring in low- and middle-income countries (LMICs), equating to 690,000 deaths/year. In 2010 an estimated 14.9 million infants were born preterm with $60 \%$ born in south Asia and sub-Saharan Africa. ${ }^{83}$ While therapeutic hypothermia has become standard of care for NE in the developed world, it is perhaps surprising, given the simplicity of the treatment that it has not been universally adopted in LMICs. A meta-analysis of therapeutic hypothermia in LMICs has shown a reduction in neonatal mortality from seven randomized controlled trials enroling a total 567 infants, although the reduction was not statistically significant. ${ }^{84}$ The data are difficult to interpret due to inconsistent inclusion and exclusion criteria and poor follow-up. The studies were heavily biased towards India, with only one RCT in Africa and none in Central and South America or countries of the former Soviet Union.

Despite inconsistency in evidence across different resource settings, there has been a gradual, if inconsistent, uptake of therapeutic hypothermia (TH) across the world. A recent survey from Brazil of 1092 professionals representing $92 \%$ of Brazilian states and federal districts found that therapeutic hypothermia was being provided in $62 \%$ of units. ${ }^{85}$ Of interest, the survey went on to show that $20 \%$ did not use any neurological score or amplitude-integrated electroencephalogram (aEEG) to assess encephalopathy; specific training for encephalopathy assessment was only provided to $19 \%$ of professionals and only $31 \%$ reported having a well-established follow-up programme for these infants.

The potential dangers of translating practice to different resource settings was highlighted by the ACT trial on the use administration of antenatal corticosteroids to mothers in threatened preterm labour. While this has clearly been shown to reduce neonatal mortality and neurodisability in high-income countries, this large cluster randomized trial across multiple LMICs showed an increase in mortality. ${ }^{86}$ Therefore, any translation of practices in the NeuroNICU model needs to be carefully assessed within different populations tailored to the needs, pathology and resources of each environment.

The neonatal environment and the developing brain With its initial focus on survival, the NICU has traditionally been modelled on conventional intensive care units. Indeed, in the early years, parents were actively discouraged from visiting their babies. The importance of family centred care was championed in the 1970 s by $\mathrm{Als}^{87}$ who created the Newborn Individualized Developmental Care and Assessment Program (NIDCAP). ${ }^{87}$ While the evidence base for NIDCAP specifically in improving neurodevelopmental outcome is limited, parents have increasingly been encouraged to take a far more active role in the care of their baby right from admission to the NICU. ${ }^{88,89}$

The harshness of the NICU is in stark contrast to the warm, dark protective in utero environment. The preterm infant is exposed to numerous painful procedures, which may directly impact on brain development. $^{90}$ The effect of the environment, such as noxious noise or excessive light, may alter short-term physiological stability and in the longer term, impede growth and development. $^{91}$ However, the evidence base for the effects of noise reduction and cycled light on neurodevelopmental outcome remains limited. ${ }^{92,93}$ Sleep-wake cycling develops in the third trimester and impaired quality and quantity of sleep on the developing brain may have a significant effect on the developing brain. $^{94}$

In designing the NICU, the debate between single rooms vs. open wards remains ongoing; the evidence from a recent systematic review and meta-analysis by van Veenendaal et al. ${ }^{95}$ reported a reduction in the incidence of sepsis and increase in breastfeeding rates in single family rooms, but no effect on neurodevelopmental outcome.

\section{CONCLUSIONS}

Neonatal intensive care has been one of the remarkable success stories in modern medicine, with dramatic improvement in survival of the smallest and sickest infants over the past 50 years. Learning from the experience of adult neurocritical care, the development of the Neuro-NICU offers real promise that the gap between survival and improved neurodevelopmental outcome can be closed. However, this requires significant investment of time, manpower and equipment, and so it is essential that prospective data is collected from large cohorts, to determine whether this development equates with improvement in outcome.

\section{ACKNOWLEDGEMENTS}

The author receives funding from NIHR Cambridge Biomedical Research Centre (www.cambridgebrc.nihr.ac.uk) and NIHR Brain Injury MedTech Co-Operative (www. brainmic.nihr.ac.uk) 


\section{AUTHOR CONTRIBUTIONS}

I can confirm that I, T.A., am the sole author of this paper, having conceived of the idea for the manuscript, undertaken the relevant literature search into the subject and written the manuscript.

\section{ADDITIONAL INFORMATION}

Competing interests: The author declares no competing interests.

Publisher's note Springer Nature remains neutral with regard to jurisdictional claims in published maps and institutional affiliations.

\section{REFERENCES}

1. Volpe, J. J. et al. Volpe's Neurology of the Newborn 6th edn (Saunders, Elsevier, Philadelphia, 2018).

2. Mangham, L. J. et al. The cost of preterm birth through childhood in England and Wales. Pediatrics 123, e312-e327 (2009).

3. Honeycutt, A. et al. Centers for Disease Control and Prevention (CDC). Economic costs associated with mental retardation, cerebral palsy, hearing loss, and vision impairment-United States, 2003. Morb. Mortal. Wkly Rep. 53, 57-59 (2004).

4. Moore, T. et al. Neurological and developmental outcome in extremely preterm children born in England in 1995 and 2006: the EPICure studies. BMJ 345, e7961 (2012).

5. Younge, N. et al. Survival and neurodevelopmental outcomes among periviable infants. N. Engl. J. Med. 376, 617-628 (2017).

6. Liu, L. et al. Global, regional and national causes of under-5 mortality in 2000-15: an updated systematic analysis with implications for the Sustainable Development Goals. Lancet 388, 3027-3035 (2016).

7. Jacobs, S. E. et al. Cooling for newborns with hypoxic-ischaemic encephalopathy. Cochrane Database Syst. Rev. 1, CD003311 (2013).

8. Smith, M. \& Menon, D. In: Masterson, G. \& Baudouin, S. eds. GPICS 2015 (ICS and FICM, London, 2015).

9. Patel, H. C. et al. Trauma Audit and Research Netwok. Trends in head injury outcome from 1989-2003 and the effect of neurosurgical care: an observational study. Lancet 366, 1538-1544 (2005).

10. Harrison, D. A. et al. Risk adjustment in neurocritical care (RAIN)-Prospective validation of risk prediction models for adult patients with acute traumatic brain injury to use to evaluate the optimum location and comparative costs of neurocritical care: a cohort study. Health Technol. Assess. 17, vii-viii (2013). 1-350.

11. Damian, M. S. et al. The effect of secular trends and specialist neurocritical care on mortality for patients with intracerebral haemorrhage, myasthenia gravis and Guillain-Barr. Syndrome admitted to critical care: an analysis of the Intensive Care National Audit \& Research Centre (ICNARC) national United Kingdom database. Intens. Care Med. 39, 1405-1412 (2013).

12. Samuels, O. et al. Impact of a dedicated neurocritical care team in treating patients with aneurysmal subarachnoid hemorrhage. Neurocrit. Care 14, 334-340 (2011).

13. Tweedie, I. Neuro-critical care versus general critical care for neurological injury: beneficial evidence. J. Neuroanaesthesiol. Crit. Care 3, 62-65 (2016).

14. Stroke Unit Trialists' Collaboration. Organised inpatient (stroke unit) care for stroke. Cochrane Database Syst. Rev. 9, CD000197 (2013).

15. The National Institute of Neurological Disorders and Stroke rt-PA Stroke Study Group. Tissue plasminogen activator for acute ischemic stroke. N. Engl. J. Med. 333, 1581-1587 (1995).

16. Fassbender, K. et al. Mobile stroke units for prehospital thrombolysis, triage and beyond: benefits and challenges. Lancet Neurol. 16, 227-237 (2017).

17. Kurinczuk, J. et al. Epidemiology of neonatal encephalopathy and hypoxicischaemic encephalopathy. Early Hum. Dev. 86, 329-338 (2010).

18. Fluss, J., Dinomais, M. \& Chabrier, S. Perinatal stroke syndromes: similarities and diversities in aetiology, outcome and management. Eur. J. Paediatr. Neurol. 23, 368-383 (2019).

19. Stoll, B. J. et al. Trends in care practices, morbidity, and mortality of extremely preterm neonates, 1993-2012. JAMA 314, 1039-1051 (2015).

20. Back, S. A. White matter injury in the preterm infant: pathology and mechanisms. Acta Neuropathol. 134, 331-349 (2017).

21. Twilhaar, E. S. et al. Cognitive outcomes of children born extremely or very preterm since the 1990 s and associated risk factors a meta-analysis and metaregression. JAMA Pediatr. 172, 361-367 (2018).

22. Guthrie, R. \& Bickel, H. The introduction of newborn screening for phenylketonuria: a personal history. Eur. J. Pediatr. 155, S4-S5 (1996).

23. Pisano, T. et al. Early and effective treatment of KCNQ2 encephalopathy. Epilepsia 56, 685-691 (2015).
24. Tseng, C.-E. et al. Verbal fluency is affected by altered brain lateralisation in adults who were born very preterm. eNeuro 6, e0274-18.2018 (2019).

25. Pape, K. E. et al. Ultrasound detection of brain damage in preterm infants. Lancet 8129, 1261-1264 (1979)

26. Cady, E. B. et al. Non-invasive investigation of cerebral metabolism in newborn infants by phosphorus nuclear magnetic resonance spectroscopy. Lancet $\mathbf{8 3 3 3}$, 1059-1062 (1983)

27. Thoresen, M. et al. Mild hypothermia after severe transient hypoxia-ischemia ameliorates delayed cerebral energy failure in the newborn piglet. Pediatr. Res. 37, 667-670 (1995).

28. Gunn, A. J. et al. Therapeutic hypothermia translates from ancient history in to practice. Pediatr. Res. 81, 202-209 (2017).

29. Watson, H. I., Shepherd, A. A., Rhodes, J. K. J. \& Andrews, P. Revisited: a systematic review of therapeutic hypothermia for adult patients following traumatic brain injury. Crit. Care Med. 46, 972-979 (2018).

30. Wyllie, J. et al. Part 11: neonatal resuscitation: 2010 International Consensus on Cardiopulmonary Resuscitation and Emergency Cardiovascular Care Science With Treatment Recommendations. Resuscitation 81(Suppl. 1), e260-e287 (2010).

31. Azzopardi, D. V. et al. Moderate hypothermia to treat perinatal asphyxia encephalopathy. N. Engl. J. Med. 361, 1349-1358 (2009).

32. Azzopardi, D. et al. Implementation and conduct of hypothermia for perinatal asphyxia encephalopathy in the UK-analysis of national data. PLOS ONE 7, e38504 (2012).

33. Chaudhary, R., Farrer, K., Broster, S., McRitchie, L. \& Austin, T. Active versus passive cooling during neonatal transport. Pediatrics 132, 841-846 (2013).

34. Glass, H. C. et al. Neurocritical care for neonates. Neurocrit. Care 12, 421-429 (2010).

35. Bashir, R. A. et al. Implemenation of a neurocritical care program: improved seizure detection and decreased antiseizure medication at discharge in neonates with hypoxic-ischemic encephalopathy. Pediatr. Neurol. 64, 38-43 (2016).

36. Harbert, M. J. et al. Impact of a neuro-intensive care service for newborns. J. Neonatal Perinat. Med. 11, 173-178 (2018).

37. Glass, H. C., Ferreiro, D. M., Rowitch, D. H. \& Shimotake, T. K. The neurointensive nursery: concept, development and insights gained. Curr. Opin. Pediatr. 31, 202-209 (2019).

38. Leotsakosi, A. et al. Standardization in patient safety: the WHO high 5 s project. J. Qual. Health Care 26, 109-116 (2014).

39. Woolf, S. H. Practice guidelines: a new reality in medicine. I. Recent developments. Arch. Intern. Med. 150, 1811-1818 (1990).

40. Maynard, D., Prior, P. F. \& Scott, D. F. Device for continuous monitoring of cerebral activity in resuscitated patients. BMJ 4, 545-546 (1969).

41. Viniker, D. A., Maynard, D. E. \& Scott, D. F. Cerebral function monitor studies in neonates. Clin. Electroencephalogr. 15, 185-192 (1984).

42. Hellstrom-Westas, L., Rosen, I. \& Swenningsen, N. W. Silent seizures in sick infants in early life. Diagnosis by continuous cerebral function monitoring. Acta Paediatr. Scand. 74, 741-748 (1985).

43. Gluckman, P. D. et al. Selective head cooling with mild systemic hypothermia after neonatal encephalopathy: multicentre randomised trial. Lancet $\mathbf{3 6 5}$, 663-670 (2005)

44. Gunn, J. K. et al. Perioperative amplitude-integrated EEG and neurodevelopment in infants with congenital heart disease. Intens. Care Med. 38, 1539-1547 (2012).

45. Olischar, M. et al. Amplitude-integrated electroencephalography in newborns with inborn errors of metabolism. Neonatology 102, 203-211 (2012).

46. Burdjalov, V. F., Baumgart, S. \& Spitzer, A. R. Cerebral function monitoring: a new scoring system for the evaluation of brain maturation in neonates. Pediatrics 112, 855-861 (2003).

47. Lloyd, R. O., O'Toole, J. M., Pavlidis, E., Filan, P. M. \& Boylan, G. B. Electrographic seizures during the early postnatal period in preterm infants. J. Pediatr. 187, 18-25 (2017).

48. Shellbaas, R. A. et al. The American Clinical Neurophysiology Society's guideline on continuous electroencephalography monitoring in neonates. J. Clin. Neurophysiol. 28, 611-617 (2011).

49. da Costa, C. S., Greisen, G. \& Austin, T. Is near-infrared spectroscopy clinically useful in the preterm infant? Arch. Dis. Child Fetal Neonatal Ed. 100, F558-F561 (2015).

50. Hyttel-Sorensen, S. et al. Cerebral near infrared spectroscopy oximetry in extremely preterm infants: phase II randomised clinical trial. BMJ 350, g7635 (2015).

51. Siegel, M. et al. Hypoxic-ischemic encephalopathy in term infants: diagnosis and prognosis evaluated by ultrasound. Radiology 152, 395-399 (1984).

52. Archer, L., Levene, M. \& Evans, D. Cerebral artery Doppler ultrasonography for prediction of outcome after perinatal asphyxia. Lancet 328, 1116-1118 (1986).

53. Boo, N. et al. Early cranial ultrasound changes as predictors of outcome during first year of life in term infants with perinatal asphyxia. J. Paediatr. Child Health 36, 363-369 (2000) 
54. Shankaran, S. et al. Neonatal magnetic resonance imaging pattern of brain injury as a biomarker of childhood outcomes following a trial of hypothermia for neonatal hypoxic-ischemic encephalopathy. J. Pediatr. 167, 987-993 (2015).

55. Lally, P. J. et al. Magnetic resonance spectroscopy assessment of brain injury after moderate hypothermia in neonatal encephalopathy: a prospective multicentre cohort study. Lancet Neurol. 18, 35-45 (2019).

56. Hintz, S. R. et al. Neuroimaging and neurodevelopmental outcome in extremely preterm infants. Pediatrics 135, e32-e42 (2015).

57. Laptook, A. R. et al. Adverse neurodevelopmental outcomes among extremely low birth weight infants with a normal head ultrasound: prevalence and antecedents. Pediatrics 115, 673-680 (2005).

58. Broitman, E. et al. Clinical data predict neurodevelopmental outcome better than head ultrasound in extremely low birth weight infants. J. Pediatr. 151, 500-505 (2007).

59. Woodward, L. J., Anderson, P. J., Austin, N. C., Howard, K. \& Inder, T. A. Neonatal MRI to predict neurodevelopmental outcomes in preterm infants. N. Engl. J. Med. 355, 685-694 (2006).

60. Edwards, A. D. et al. Effect of MRI on preterm infants and their families: a randomised trial with nested diagnostic and economic evaluation. Arch. Dis. Child Fetal Neonatal Ed. 103, F15-F21 (2018).

61. Peloquin, S., Carley, A., Bonifacio, S. L. \& Glass, H. C. The neurointensive care nursery and evolving roles for nursing. Neonatal Netw. 35, 87-94 (2016).

62. Smyser, C. D. et al. Fellowship training in the emerging fields if fetal-neonatal neurology and neonatal neurocritical care. Pediatr. Neurol. 63, 39-44 (2016).

63. Mulkey, S. B. \& Swearingen, C. J. Advancing neurologic care in the neonatal intensive care unit with a neonatal neurologist. J. Child Neurol. 29, 31-35 (2014).

64. Wietstock, S. O. et al. Continuous video electroencephalographic (EEG) monitoring for electrographic seizure diagnosis in neonates: a single-center study. J. Child Neurol. 31, 328-332 (2016).

65. Wietstock, S. O. et al. Neonatal neurocritical care service is associated with decreased administration of seizure medication. J. Child Neurol. 30, 1135-1141 (2015).

66. Rhodes, A. et al. Surviving sepsis campaign: international guidelines for management of sepsis and septic shock: 2016. Intens. Care Med. 43, 304-377 (2017).

67. Adams-Chapman, I. Necrotizing enterocolitis and neurodevelopmental outcome. Clin. Perinatol. 45, 453-466 (2018).

68. Chiriboga, N. et al. Successful implementation of an intracranial (ICH) bundle in reducing severe ICH: a quality improvement project. J. Perinatol. 39, 143-151 (2019).

69. Chollat, C., Sentihes, L. \& Marret, S. Protection of brain development by antenatal magnesium sulphate for infants born preterm. Dev. Med. Child Neurol. 61, 25-30 (2019).

70. Azzopardi, D. et al. Moderate hypothermia within $6 \mathrm{~h}$ of birth plus inhaled xenon versus moderate hypothermia alone after birth asphyxia (TOBY-Xe): a proof-ofconcept, open-label, randomised controlled trial. Lancet Neurol. 15, 145-153 (2016).

71. Juul, S. E. et al. High-dose erythropoietin for asphyxia and encephalopathy (HEAL): a randomized controlled trial: background, aims, and study protocol. Neonatology 113, 331-338 (2018).

72. Maiwald, C. A. et al. Effect of allopurinol in addition to hypothermia treatment in neonates for hypoxic-ischemic brain injury on neurocognitive outcome (ALBINO): study protocol of a blinded randomized placebo-controlled parallel group multicentre trial for superiority (phase III). BMC Pediatr. 19, 210 (2019).

73. Chalak, L., Latremouille, S., Mir, I., Sanchez, P. J. \& Sant'Anna, G. A review of the conundrum of mild hypoxic-ischemic encephalopathy: current challenges and moving forward. Early Hum. Dev. 120, 88-94 (2018).

74. Farnaes, L. et al. Rapid whole-genome sequencing decreases infant morbidity and cost of hospitalisation. NPJ Genom. Med. 3, 10 (2018).
75. Elliott, A. M. et al. RAPIDOMICS: rapid genome-wide sequencing in a neonatal intensive care unit-successes and challenges. Eur. J. Pediatr. 178, 1207-1218 (2019).

76. French, C. E. et al. Whole genome sequencing reveals that genetic conditions are frequent in intensively ill children. Intens. Care Med. 45, 627-636 (2019).

77. Wilkinson, D. J. C., Barnett, C., Savulescu, J. \& Newson, A. J. Genomic intensive care: should we perform genome testing in critically ill newborns? Arch. Dis. Child Fetal Neonatal Ed. 101, F94-F98 (2016).

78. Thewissen, L. et al. Measuring near-infrared spectroscopy derived cerebral autoregulation in neonates: from research tool toward bedside multimodal monitoring. Front. Pediatr. 6, 117 (2018).

79. Temko, A. et al. Toward a personalized real-time diagnosis in neonatal seizure detection. IEEE J. Transl. Eng. Health Med. 5, 2800414 (2017).

80. Joshi, R. et al. Pattern discovery in critical alarms originating from neonates under intensive care. Physiol. Meas. 37, 564-579 (2016).

81. Vellido, A., Ribas, V., Morales, C., Ruiz Sanmartín, A. \& Ruiz Rodríguez, J. C. Machine learning in critical care: state-of-the-art and a sepsis case study. Biomed. Eng. Online 17, 135 (2018).

82. Sortica da Costa, C. et al. Complexity of brain signals is associated with outcome in preterm infants. J. Cereb. Blood Flow Metab. 37, 3368-3379 (2017).

83. Blencowe, $\mathrm{H}$. et al. National, regional, and worldwide estimates of preterm birth rates in the year 2010 with time trends since 1990 for selected countries: a systematic analysis and implications. Lancet 379, 2162-2172 (2012).

84. Pauliah, S. S., Shankaran, S., Wade, A., Cady, E. B. \& Thayyil, S. Therapeutic hypothermia for neonatal encephalopathy in low- and middle-income countries: a systematic review and meta-analysis. PLOS ONE 8, e58834 (2013).

85. Variane, G. F. et al. Therapeutic hypothermia in Brazil: a multiprofessional national survey. Am. J. Perinatol. https://doi.org/10.1055/s-0038-1676052 (2018).

86. Althabe, F. et al. A population-based, multifaceted strategy to implement antenatal corticosteroid treatment versus standard care for the reduction of neonatal mortality due to preterm birth in low-income and middle-income countries: the ACT cluster randomised trial. Lancet 385, 629-639 (2015).

87. Als, $\mathrm{H}$. et al. Individualised behavioural and environmental care for the very low birth weight preterm infant at high risk for bronchopulmonary dysplasia: meonatal intensive care unit and developmental outcome. Pediatrics 78, 1123-1132 (1986).

88. Ohlsson, A. \& Jacobs, S. E. NIDCAP: a systematic review and meta-analyses of randomized controlled trials. Pediatrics 131, e881-e893 (2013).

89. Franck, L. S. \& O'Brien K. The evolution of family-centred care: from supporting parent-delivered interventions to a model of family integrated care. Birth Defects Res. https://doi.org/10.1002/bdr2.1521 (2019).

90. Brummelte, S. et al. Procedural pain and brain development in premature newborns. Ann. Neurol. 71, 385-396 (2012).

91. Santos, J., Pearcea, S. E. \& Stroustrupa, A. Impact of hospital-based environmental exposures on neurodevelopmental outcomes of preterm infants. Curr. Opin. Pediatr. 27, 254-260 (2015).

92. Almadhoob, A. \& Ohlsson, A. Sound reduction management in the neonatal intensive care unit for preterm or very low birth weight infants. Cochrane Database Syst. Rev. 1, CD010333 (2015).

93. Morag, I. \& Ohlsson, A. Cycled light in the intensive care unit for preterm and low birth weight infants. Cochrane Database Syst. Rev. 8, CD006982 (2016).

94. Bennet, L., Walker, D. W. \& Horne, R. S. C. Waking up too early-the consequences of preterm birth on sleep development. J. Physiol. 596, 5687-5708 (2018).

95. Van Veenendaal, N. R. et al. Hospitalising preterm infants in single family rooms versus open bay units: a systematic review and meta-analysis. Lancet Child Adolesc. Health 3, 147-157 (2019). 